\title{
A review of X-ray spectral evolution in Crab pulsar
}

\author{
M. Vivekanand ${ }^{\star}$ \\ National Center for Radio Astrophysics, TIFR, Pune University Campus, PO Box 3, Ganeshkhind, Pune 411007, India \\ Received 22 April 2002 / Accepted 17 June 2002

\begin{abstract}
Pravdo et al. (1997) claimed that the phase resolved x-ray spectrum in Crab pulsar (PSR B0531+21) shows a spectral hardening at the leading edge of the first peak of its integrated profile (IP); this was a new and unexpected result. This article reanalyzes their data, as well as some other related data, and argues that the spectrum is as likely to be unvarying (i.e., neither hardening nor softening).
\end{abstract}

Key words. pulsars - individual: PSR B0531+21 - X-ray: stars - phase resolved spectra

\section{Introduction}

Pravdo et al. (1997; henceforth PAH) studied the power law spectrum of Crab pulsar as a function of phase within its IP, in the energy range $5 \mathrm{KeV}$ to $60 \mathrm{KeV}$, using $\approx 86000$ periods of data obtained by the PCA detector aboard the RXTE X-ray observatory. Their photon arrival times had a resolution of $250 \mu \mathrm{s}$, allowing them to obtain the spectrum in 60 on-pulse phase bins, each of duration $\approx 335 \mu$ s, out of a total of 100 phase bins within the IP. Their main conclusions are "(1) the spectrum softens (i.e., the power law index increases) starting at the leading edge of the first peak until the intensity maximum of the first peak; (2) "the spectrum hardens in the inter peak region; and (3) the spectrum softens throughout the second peak". They point out that item (1) above is unexpected on the basis of existing theoretical models. They also point out that the power law index $\alpha$ varying as the shape of first peak of the IP may be an important clue for understanding the details of the high energy emission of the Crab pulsar. Moreover, the increase in $\alpha$ before the leading edge of the first peak of the IP coincides with the position of the radio precursor emission (Smith 1986; Lundgren et al. 1995; Moffet \& Hankins 1996). All this adds to the significance of item (1) above.

This paper reanalyzes their data, as well as some other RXTE data that have better time resolution, and sufficient energy resolution within similar energy range. This paper argues that the spectral hardening is a weak effect in terms of the overall spectral variation across the IP; the spectrum is as likely to be unvarying in the leading edge of the first peak of the IP.

For each data file, obtained in the EVENT mode (XTE_SE), the Good Time Intervals (GTI) were obtained by using the MAKETIME tool on the corresponding XTE filter file; the selection criterion were (a) pointing offset less than $0.02^{\circ}$, (b) elevation greater than $10^{\circ}$, (c) all five PCUs to be switched on, and time since passage of RXTE satellite through the South

\footnotetext{
* e-mail:vivek@ncra.tifr.res.in
}

Atlantic Anomaly (TIME_SINCE_SAA) to be greater than $30^{\circ}$ or less than $0^{\circ}$. Next the MGTIME tool was used to merge these GTIs with those in the second extension of each data file, with the AND option, to produce a final GTI file corresponding to each data file. These data/GTI file pairs were then input to the FASEBIN tool, along with the orbit file of the day, to obtain the phase resolved spectrum of Crab pulsar in 100 phase bins within the period. In the output of FASEBIN, the Crab nebula background was subtracted using an off-pulse phase range of 0.1 , using the FBSUB tool.

Table 1 lists some information about the data analyzed in this paper. The data analyzed by PAH is FS37_463ca70463d5a6, observed on 1996 May 2. They ignored the other two files observed on the same day, for "dead-time and rate considerations", although they state in their appendix that "these $8 \mathrm{~s}$ data are usable for spectral analysis in principle, since the deadtime is spectrally independent". The rest of the data was chosen from the RXTE public data archive using the criterion: (1) the number of usable energy channels within $5 \mathrm{keV}$ to $60 \mathrm{keV}$ must be at least 10 , for proper fitting of the power law spectrum, and (2) the time resolution should be much smaller than the width of each phase bin (which is $\approx 335 \mu \mathrm{s}$ ). PAH analyze data whose accuracy is comparable to the width of their phase bins; ideally the former should be much smaller than the latter. A fourth data file in ObsID 10202, and data in ObsID 40090, had the best time resolution $(2 \mu \mathrm{s})$, but $\leq 8$ usable energy channels.

The output of the FASEBIN tool is the required phase resolved spectrum. This tool uses two parameters that can potentially affect further analysis - the Crab pulsar period $P$ for the epoch of observation, and the time offset ROFF (Col. 7 of Table 1) with respect to the radio ephemeris. The former is automatically obtained from the pulsar catalog, while the latter has to be inserted by hand. Now, the $P$ of epoch for Crab pulsar can be different from the catalog determined value owing to its glitching and timing activity; and an error of 3.5 nano second in the period used for folding can cause a total drift of 1 phase 
Table 1. Columns 1-3 contain the date of observation, the ObsID of RXTE, and the name of the event mode data file. Column 4 contains the total duration of observation in seconds; this is obtained by summing the time intervals in the GTI file. In principle this is also supposed to be the time (divided by 100) found in the EXPOSURE column of the phase resolved spectrum; for unknown reasons this is not true for the ObsID 10204 data, but is true for the rest of the data. Column 5 contains the number of energy channels available within the energy range analyzed; this is $5.2 \mathrm{keV}$ to $59.1 \mathrm{keV}$ for ObsIDs 10202 and 10204, and 18.2 to $54.9 \mathrm{keV}$ for ObsID 10203 (in Vivekanand 2001a and 2001b, which analyze data of ObsID 10203, the above energy range was wrongly stated to be $13.3 \mathrm{keV}$ to $58.4 \mathrm{keV}$ ). Column 6 contains the time resolution of the data in micro seconds $(\mu \mathrm{s})$. Column 7 contains the offset time in seconds to be given to the FASEBIN tool. The last column contains the relative shift of the IPs of each data file, with respect to the IP of the data file FS37_463ca70-463d5a6, in units of pulsar phase; for comparison, the width of each phase bin is 0.01 .

\begin{tabular}{cccccccc}
\hline \hline DATE & ID & FILE & EXP & CHN & $\tau$ & ROFF & SHIFT \\
\hline \hline 1996 Apr. 18 & 10202 & FS37_451ee10-451f318 & 193 & 10 & 30 & 0.02105 & -0.0007 \\
& & FS37_451f6b0-451fc00 & 183 & 10 & 30 & 0.02105 & -0.0004 \\
1996 Apr. 20 & & FS37_4536db0-4537a88 & 497 & 10 & 30 & 0.02105 & -0.0005 \\
1996 May 2 & \multirow{2}{*}{10204} & FS37_463b300-463bf18 & 611 & 97 & 250 & 0.02110 & 0.0 \\
& & FS37_463ca70-463d5a6 & 2014 & 97 & 250 & 0.02110 & 0.0 \\
& & FS37_463e1e0-463eba8 & 464 & 97 & 250 & 0.02110 & 0.0 \\
1996 Aug. 23 & \multirow{2}{*}{10203} & FS3f_4f83ae0-4f84862 & 3310 & 10 & 4 & 0.02090 & -0.0003 \\
& & FS3f_4f851d0-4f85ee1 & 3214 & 10 & 4 & 0.02090 & -0.0003 \\
& & FS3f_4f86850-4f87561 & 3214 & 10 & 4 & 0.02091 & -0.0004 \\
& & FS3f_4f87ed0-4f88be1 & 3214 & 10 & 4 & 0.02093 & -0.0004 \\
\hline
\end{tabular}

bin in one of the longer data files in Table 1. Next, the data are obtained not in one contiguous data file, but in several files, each observed at different epochs. Therefore the correct time offset ROFF for each file is crucial to align in phase the data in those files; otherwise the $i$ th (say) phase bin in each file might correspond to different true phase bins within the pulsar period.

To avoid these problems, two things were done. First, the ASCII version of the photon counts were obtained from the FITS version of each phase resolved spectrum. An IP was formed from this data, combining all energy channels. This was cross correlated with a standard IP (that of data file 10204:FS37_463ca70-463d5a6, which itself was first suitably shifted in phase for centering it). Any significant phase shift detected was added to ROFF, and the FASEBIN tool was run once again. In this iterative manner it was ensured that the residual phase shift (Col. 8 of Table 1 ) is much less than the width of a phase bin (0.01).

Next, arrival times of photons in each data file above were converted to the solar system barycenter system (TDB) as described in Vivekanand (2001a, 2001b), using the new FAXBARY tool (improved version in HEASOFT 5.1). Then the DECODEEVT tool was used to obtained the TDB times of each photon event, and its energy channel. From the ASCII version of this data, IPs were formed of the first and second halves of each data file above. These two were then cross correlated. Their relative phase shift was sufficiently small to ensure that the period used was sufficiently accurate for the current purpose (see Vivekanand 2001a).

Penultimately, the response matrix for each data file was obtained using the PCARSP tool. Since the phase resolved spectrum output by FASEBIN is incompatible with PCARSP, a non-phase resolved spectrum was obtained for each data file using the tools SEFILTER and SEEXTRCT, using the corresponding GTI file.

Finally, the background subtracted phase resolved spectra were analyzed using the XSPEC tool. The spectrum was modeled as the power law $\mathrm{d} N / \mathrm{d} E=\beta E^{-\alpha}$ (photons per $\mathrm{keV}$ ), where $\beta$ is the normalization constant and $\alpha$ is the power law index. Only energy channels lying between $2 \mathrm{keV}$ and $60 \mathrm{keV}$ were considered, since this is the RXTE/PCA energy calibrated range. The channels to ignore were obtained by looking at the corresponding response matrices using FDUMP.

\section{Reanalysis of data of Pravdo et al. (1997)}

Figure 1 shows the result of fitting, to the data of $\mathrm{PAH}$, the power law model spectrum to 66 of the 100 phase bins, that are centered on the Crab pulsar X-ray emission. The top panel of Fig. 1 shows the background subtracted Crab pulsar emission, which is in agreement with their profile in their Fig. 1.

The middle panel of Fig. 1 shows the variation of $\beta$ with pulse phase. This is in qualitative agreement with the $\beta$ values tabulated by PAH; but these are systematically higher by the factor $\approx 1.5$. This author does not understand this factor, and neither do the people at the XTE Help Desk, who are addressing the problem. The current suspicion is that the total duration of observation (contained in the EXPOSURE column of the phase resolved spectrum) is erroneous for data of this ObsID; For this file the EXPOSURE is indicated to be 2870 s, while it is actually $2014 \mathrm{~s}$ (Table 1). Please note that this problem exists for this ObsID only. However, internal consistency has been verified by integrating, at several phases within the IP, the derived power law within a given range of energies, and comparing this with the corresponding result of the FLUX command in XSPEC.

The last panel of Fig. 1 shows the variation of $\alpha$ with pulse phase. Once again these agree qualitatively very well with the $\alpha$ values of PAH. The overall behavior is very similar, although there are differences in detail.

Figure 3 of PAH shows spectra at two phases within the IP, with the model spectrum superimposed upon the data. 


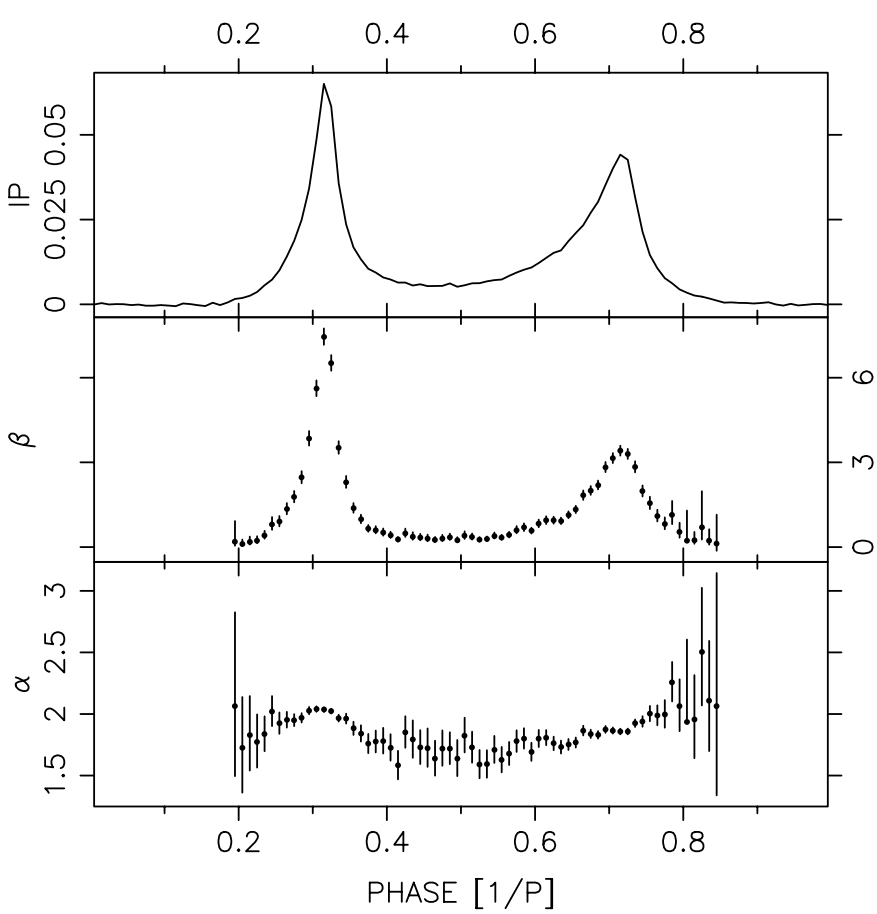

Fig. 1. Reanalysis of the data file FS37_463ca70-463d5a6, ObsID 10204, observed on 1996 May 2 (Pravdo et al. 1997). Top panel: IP of Crab pulsar. The Crab nebula emission has been subtracted by using FBSUB and the two phase ranges $0.01-0.05$, and 0.96-1.00; the area under the curve has been normalized to 1.0. Middle panel: the normalization constant $\beta$. Bottom panel: the power law index $\alpha$. In the lower two panels, the dots represent the mean values, while the vertical bars represent the limits at the $90 \%$ confidence level, as derived by XSPEC. The range of energy used is $5.2 \mathrm{keV}$ to $59.1 \mathrm{keV}$. The fit at phase 0.81 is dubious.

This figure was also reproduced in the current analysis, and there is very good qualitative agreement between the two plots.

The normalized $\chi_{\mathrm{d}}^{2}$ (i.e., $\chi^{2}$ per degree of freedom) is distributed similarly in this work and that of PAH. In the current work $\chi_{\mathrm{d}}^{2}$ lies between 0.7 and 1.4 in the 66 phase bins in which spectral estimation was possible. In PAH the range is exactly the same in the 60 phase bins analyzed. In the current work 25 of the $66 \chi_{\mathrm{d}}^{2}$ have values $\geq 1.1$, while the corresponding numbers in PAH are 30 out of 60 . The number of $\chi_{\mathrm{d}}^{2} \geq 1.2$ in the two works is the same, viz., 14.

It is therefore concluded that this analysis broadly reproduces the results of PAH.

Some minor differences exist between this analysis and that of PAH. In this analysis, a range of 0.1 in phase is used for nebular background subtraction (0.01-0.05, and 0.96-1.00), while the latter use a range of 0.3 . Next, this work quotes the $90 \%$ confidence limits on $\alpha$ and $\beta$, while the latter quote the one standard deviation limits. Next, the boundaries of the phase bins will not match exactly in the two cases, since the centering of the Crab emission within the IP is done differently (in fact, arbitrarily) in the two cases. In this analysis the exact energy range chosen for analysis is $5.2 \mathrm{keV}$ to $59.1 \mathrm{keV}$ (ignored energy channels 98 on wards); the corresponding numbers have not been mentioned by PAH. For example, if they included the next energy channel, their energy range would go up to $60.4 \mathrm{keV}$.

Other differences can also exist. For example, PAH do not mention how they obtained their GTIs. If they did not choose the selection based on TIME_SINCE_SAA, which is apparently often done to maximize scarce data, it will make a difference, although very small, to the amount of data retained for analysis.

Given these actual and possible differences, one does not expect the current results to match identically with those of PAH. So Fig. 1 will be taken as a qualitative confirmation of their broad results. One can see from this figure why they thought that there was spectral hardening at the leading edge of the first peak of the IP (phase range 0.19-0.32). If one ignores the data at phases 0.19 and 0.24 , one can discern a clear hardening of the spectrum (i.e., decrease in $\alpha$ ) at earlier phases with respect to the first peak (which falls at phase 0.32 in Fig. 1). PAH do not do any quantitative analysis in this regard, presumably because of the non-Gaussian nature of the distribution of errors.

However, one can notice that most of the visual effect of spectral hardening comes from the the four data at phases 0.20 0.23 ; unfortunately these have much higher error bars. The four data from phases $0.25-0.28$ do not show such dramatic spectral hardening; they could even be consistent with a flat distribution.

This section will be concluded with the inspiration that in Fig. 1 there is certainly a visual case for spectral hardening at the leading edge of the first peak of the X-ray IP of Crab pulsar, as noticed by PAH in the same data, not withstanding the minor differences between the two works.

In the next section it will be argued that such an inspiration could be misleading.

\section{Analysis of data that Pravdo et al. (1997) ignored}

Figure 2 displays the IP and the $\alpha$ for the other two data files of ObsID 10204, which were ignored by PAH. The method of analysis is identical to that described earlier. The IP is almost exactly the same as in Fig. 1, and broadly $\alpha$ varies with phase as in Fig. 1. The normalized $\chi_{\mathrm{d}}^{2}$ lies between 0.8 and 1.3 in the 66 phase bins in which spectral estimation was possible; 29 of these $\chi_{\mathrm{d}}^{2}$ have values $\geq 1.1$, while 13 of them have values $\geq 1.2$. Therefore the $\chi_{\mathrm{d}}^{2}$ in Fig. 2 appears to be distributed as in Fig. 1, as well as in PAH. The total duration of observation in Fig. 2 is more than half of that of Fig. 1, so one can certainly compare the $\alpha$ variation in the two figures.

In Fig. 2 there is hardly any spectral hardening in the phase range $0.19-0.32$. Now one has to ignore the data at three phases $(0.19,0.20$ and 0.22$)$ to discern the spectral hardening seen in Fig. 1. However, the $\chi_{\mathrm{d}}^{2}$ for these three data are 1.25, 1.10 and 1.15 ; so at least the latter two data are certainly well determined. What is most significant is the almost flat $\alpha$ distribution in the phase range $0.26-0.32$. This contrasts with the bump seen in the bottom panel of Fig. 1 in the same phase range. In fact, if one were allowed to disregard the single $\alpha$ at phase 0.29 in Fig. 2, then one can probably discern a trough, instead of a bump, in the phase range $0.26-0.32$ ! If one ignores none of the $\alpha$ below phase 0.32 in Fig. 2, then there appears to be more of 


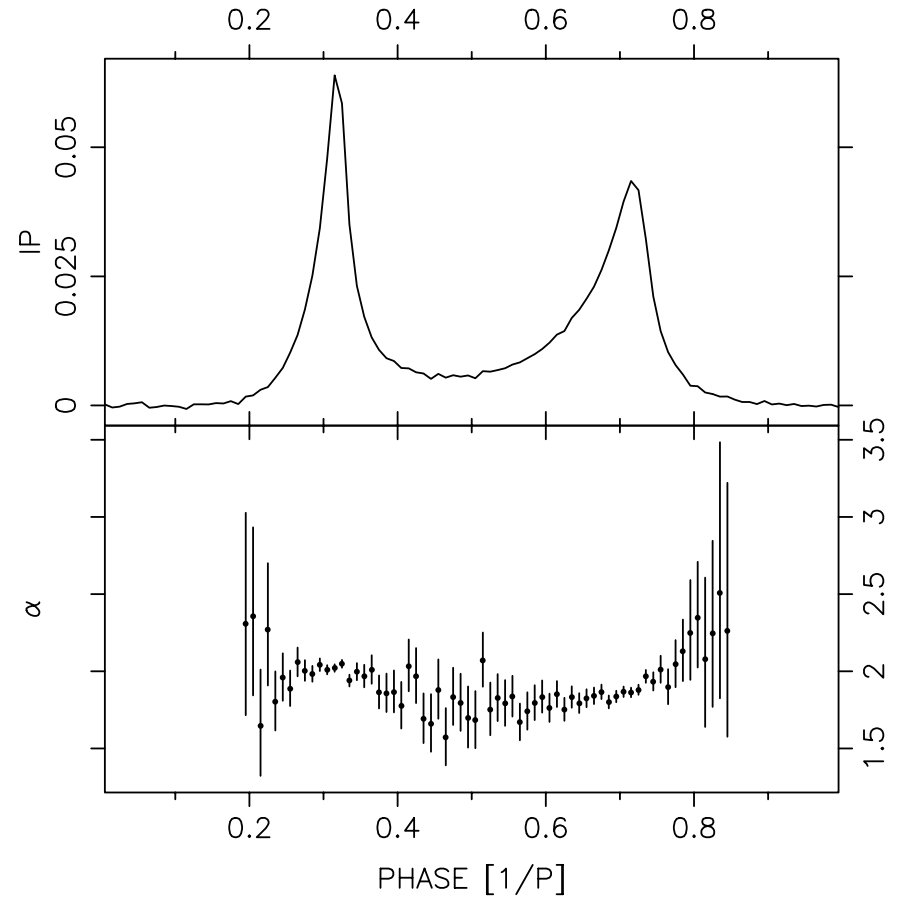

Fig. 2. Analysis of data files FS37_463b300-463bf18 and FS37_463e1e0-463eba8, ObsID 10204, observed on 1996 May 2 (this data was ignored by Pravdo et al. 1997). Top panel: IP of Crab pulsar. The Crab nebula emission has been subtracted by using FBSUB and the two phase ranges 0.01-0.05, and 0.96-1.00; the area under the curve has been normalized to 1.0. Bottom panel: the power law index $\alpha$. The dots represent the mean values, while the vertical bars represent the limits at the $90 \%$ confidence level, as derived by XSPEC. The range of energy used is $5.2 \mathrm{keV}$ to $59.1 \mathrm{keV}$.

a case for a flat $\alpha$ distribution, rather than it decreasing with decreasing phase.

It is unfortunate that one is unable to do a quantitative analysis like the standard $\chi^{2}$, due to the non-Gaussian nature of the errors. However, at the level of the visual analysis done by PAH, this section concludes that the data they ignored does not appear to show the spectral hardening that they claim. The data of Fig. 2 have been analyzed in the same manner as that in Fig. 1. So whatever may be the differences between Fig. 1 here and the results of PAH, one expects consistency between Figs. 1 and 2 of this paper. It is predicted that if PAH were to analyze the data they have ignored, in the same manner as in PAH, they will reproduce a figure similar to Fig. 2 here.

\section{Analysis of other data}

\subsection{ObsID 10202}

Figure 3 shows the results for three files of ObsID 10202. The reason they were not combined is the following. First, they belong to different sets of observations (10202-02-01-00 and 10202-02-02-00); from their response files one notices small differences in the labeling of their energy channels. Second, there was some technical problem in obtaining the response matrix for the data file FS37_451f6b0-451fc00 (by the method mentioned in Sect. 1). So it was obtained by not using the

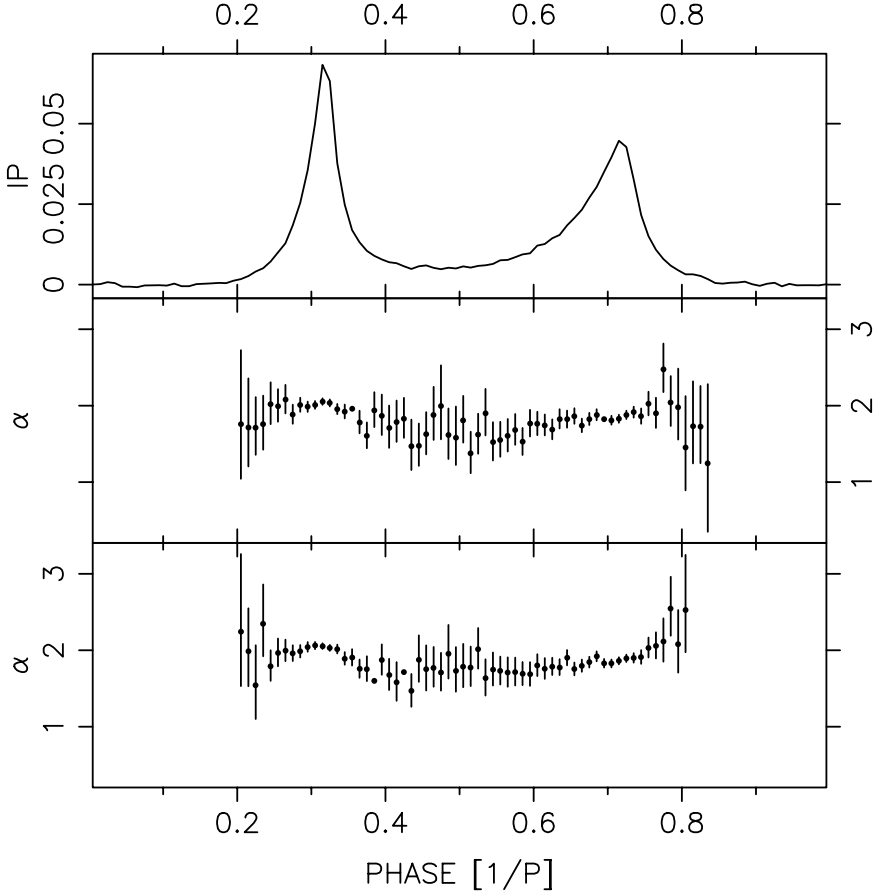

Fig. 3. Analysis of data of ObsID 10202. Top panel: same as the top panel in Fig. 1 but for data files FS37_451ee10-451f318 and FS37_451f6b0-451fc00. Middle panel: same as the bottom panel in Fig. 2, but for data files FS37_451ee10-451f318 and FS37_451f6b0451fc00. Bottom panel: same as the bottom panel in Fig. 2, but for data file FS37_4536db0-4537a88. The range of energy used is $5.2 \mathrm{keV}$ to $59.1 \mathrm{keV}$.

attitude file. It was verified that the numerical values in each column of this response matrix are very similar to those in the response matrix for the other file (FS37_451ee10-451f318) in this observation set; the maximum difference was $0.3 \%$.

In the bottom panel of Fig. 3, $\alpha$ appears to behave as in Fig. 2, rather than as in Fig. 1. The bump in the phase range $0.26-0.32$ is less prominent than in Fig. 1, and $\alpha$ at the smallest phases appears to be higher rather than lower. Even if one ignores the data at phase 0.20 , which has a high $\chi_{\mathrm{d}}^{2}$ of 1.9 , the spectral hardening claimed by PAH is weak, at best. The $\chi_{\mathrm{d}}^{2}$ vales in this panel range from 0.2 to 3.0 ; the numbers lying $\geq 1.1$ and $\geq 1.2$ are 27 and 21 , respectively, out of the 61 phase bins analyzed. This is similar to the situation in Figs. 1 and 2.

The spectral hardening is weaker, and probably nonexistent, in the middle panel of Fig. 3. The $\chi_{\mathrm{d}}^{2}$ distribution in this panel is similar to other distributions.

\subsection{ObsID 10203}

Figure 4 shows the results for four data files of ObsID 10203; their combined observing time is much larger than that of any other set in Table 1 . The individual $\chi_{\mathrm{d}}^{2}$ of the first eight data from phases 0.19 to 0.26 does not exceed 1.16; so these $\alpha$ values are very reliable. For the 65 phases analyzed in this figure, the $\chi_{\mathrm{d}}^{2}$ range from 0.6 to $2.1 ; 24$ of these have values $\geq 1.1$ while 14 of these values $\geq 1$. . If one could ignore the $\alpha$ at phase 0.21 , one might argue that a strong case exists for spectral hardening; but if one could ignore the first two $\alpha$ also, then the case 


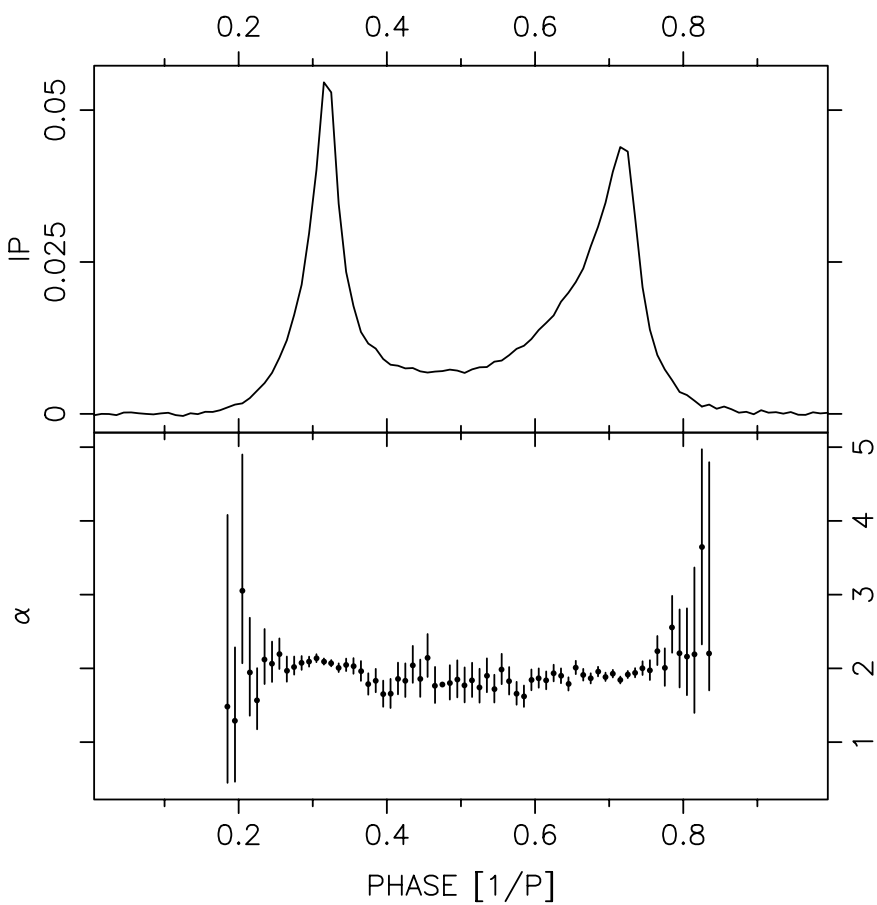

Fig. 4. Analysis of data of ObsID 10203. Top panel: same as the top panel in Fig. 2 but for the four data files of this ObsID. Bottom panel: same as the bottom panel in Fig. 2, but for the four data files of this ObsID. The range of energy used is $18.2 \mathrm{keV}$ to $54.9 \mathrm{keV}$.

becomes much weaker. If one could ignore the first five data (phases 0.19-0.23) then the case becomes really weak. Even if one ignores the first eight data, it is not clear how strong the case is for spectral hardening, if one takes into account the error bars on the four data at phases $0.27-0.30$.

\section{Discussion}

This paper therefore concludes that the spectral hardening noticed by PAH, at the leading edge of the first peak of Crab pulsar's X-ray IP, is not a strong result, although PAH were probably not unjustified in arriving at such a conclusion from their own analysis. Other data, particularly that which was part of the data set of PAH but which they ignored, indicates that a constant $\alpha$ is as likely a solution in contrast to a decreasing $\alpha$.

Apparently the above spectral hardening has also been noticed by Massaro et al. (2000) in the energy range $1.6 \mathrm{keV}$ to $300 \mathrm{keV}$, using the BeppoSAX observatory. They claim in their discussion that regarding the first peak of the IP, they have "confirmed... that both the leading and trailing edges have harder spectra than the central bins". In particular they note that in their Figs. 3c and 3d, "the central bins" of the first peak "have a softer spectrum than the wings"; these figures are similar to the bottom panel of Fig. 2 here, but for energy ranges $10 \mathrm{keV}$ to $34 \mathrm{keV}$, and $15 \mathrm{keV}$ to $300 \mathrm{keV}$, respectively. However, a closer look at these two figures of Massaro et al. (2000) indicates that this result may be be non-existent in their Fig. 3d; $\alpha$ appears to be more or less constant at phases below that of the first peak of the IP. Their Fig. 3c certainly contains what might be interpreted as the spectral hardening noticed by PAH. If one were to take both these results seriously, one might be forced to the interpretation that the spectral hardening, if at all it exists in the BeppoSAX data, is likely to be a phenomenon confined to a narrow energy range (10 $\mathrm{keV}$ to $34 \mathrm{keV})$.

Figure $3 \mathrm{~b}$ of Massaro et al. (2000), energy range $1.6 \mathrm{keV}$ to $10 \mathrm{keV}$, shows that $\alpha$ may be constant at those phases where it decreases the most in their Fig. 3c; and it shows a small bump where it is more or less constant in their Fig. 3c. Finally their Fig. $4 \mathrm{a}$ (15 keV to $80 \mathrm{keV}$ range) appears to show a nearly unvarying $\alpha$ at the leading edge of the first IP peak. It therefore appears that the results of Massaro et al. (2000) are also indicating the kind of confusion that has been presented in this paper, regarding the spectral hardening claimed by $\mathrm{PAH}$.

At this stage one can certainly not rule out the spectral hardening claimed by $\mathrm{PAH}$, particularly since another independent instrument claims to have noticed the same. However, one should also keep in mind the divergence of results in this regard, and also not rule out the possibility that $\alpha$ may be more or less constant in the leading edge of the first peak of the IP. This is particularly important in view of (a) the lack of quantitative analysis in this regard, and (b) a recent theoretical study prefers a monotonically increasing $\alpha$ at smaller phases within the IP, and neither a constant nor a decreasing $\alpha$ (Zhang \& Cheng 2002). To accommodate the spectral hardening of PAH, they need to invoke emission other than synchrotron radiation from secondary pairs.

Pravdo et al. (1997) claim that phase resolved X-ray spectrum in Crab pulsar "softens throughout the second peak" of the IP (their Fig. 1). However Fig. 1 of this paper shows that this is also a weak effect, as do Fig. 4 and the bottom panel of Fig. 3; in these figures the spectrum could be unvarying in the trailing edge of the second peak also. The above claim of PAH appears to be more evident in Fig. 2 of this paper. However, in the second panel of Fig. 3, the spectrum appears to harden in thetrailing edge of the second peak, opposite to the claim of PAH. Keeping in mind the lack of quantitative analysis, and the confusing visual results from different data sets, one can not rule out a constant $\alpha$ in the trailing edge of the second peak of the X-ray IP of Crab pulsar.

Acknowledgements. I thank Teresa Mineo and the anonymous referee for useful suggestions. This research has made use of (a) High Energy Astrophysics Science Archive Research Center's (HEASARC) facilities such as their public data archive, and their FTOOLS software, and (b) NASA's Astrophysics Data System (ADS) Bibliographic Services.

\section{References}

Lundgren, S. C., Cordes, J. M., Ulmer, M., et al. 1995, ApJ, 453, 433 Massaro, E., Cusumano, G., Litterio, M., \& Mineo, T. 2000, A\&A, 361,695

Moffet, D. A., \& Hankins, T. H. 1996, ApJ, 468, 779

Pravdo, S. H., Angelini, L., \& Harding, A. K. 1997, ApJ, 491, 808 (PAH)

Smith, F. G. 1986, MNRAS, 219, 729

Vivekanand, M. 2001, A\&A, 373, 236

Vivekanand, M. 2001, A\&A, 376, 580

Zhang, L., \& Cheng, K. S. 2002, ApJ, 569, 872 